\title{
46. DATA REPORT: LATE PLIOCENE DISCOASTER ABUNDANCES FROM HOLE 806C ${ }^{1}$
}

\author{
Jan Backman ${ }^{2}$ and Alexander Chepstow-Lusty ${ }^{3}$
}

\section{INTRODUCTION}

Members of the calcareous nannofossil genus Discoaster have been used extensively to subdivide Tertiary deep-sea sediments into biostratigraphic zones or subzones (e.g., Martini, 1971; Bukry, 1973). Haq and Lohmann (1976) mapped biogeographic migrations of this group through time and over latitude. They suggested that expansions and contractions of Discoaster-dominated assemblages across latitudes reflect sea-surface temperature changes. Subsequently, late Pliocene Discoaster species were counted at closely spaced sample intervals from various Atlantic sites (Backman et al., 1986; Backman and Pestiaux, 1987; Chepstow-Lusty et al., 1989, 1991), and Indian Ocean as well as Pacific Ocean sites (Chepstow-Lusty, 1990). In addition to the biostratigraphic information revealing positions and the precision by which the different late Pliocene Discoaster species can be determined, these studies also demonstrated that discoasters strongly fluctuate in abundance as a function of time. These abundance variations occur in equatorial as well as temperate temperature regimes, and show periodicities that reflect orbital frequencies. Chepstow-Lusty et al. (1989, 1991) also suggested that the oscillating abundances partly represent productivity pressure, because discoasters tend to show low abundances under high productivity conditions and vice versa.

In the Pacific Ocean, counts showing late Pliocene Discoaster abundances exist from three sites, namely Ocean Drilling Program (ODP) Site 677 in the eastern equatorial upwelling region, Core V28-179 from the central equatorial region, and Core V32-127 from the mid-latitude Hess Rise. The two Vema cores are condensed and show sedimentation rates below $0.5 \mathrm{~cm} / 1000 \mathrm{yr}$, thus offering a poorly resolved stratigraphy. Hole $806 \mathrm{C}$ from the Ontong Java Plateau provided an opportunity to establish a highly resolved Discoaster record from the western extreme of the equatorial Pacific under an environmental setting that differed from ODP Site 677 by being less influenced by intense upwelling. The Discoaster counting technique is described by Backman and Shackleton (1983).

\section{RESULTS}

\section{Discoaster brouweri and Discoaster triradiatus}

Abundances of $D$. brouweri and $D$. triradiatus as a function of sub-bottom depth are shown in Figure 1. The former species is clearly dominant in the interval investigated. The abundance plot of $D$. brouweri shows two conspicuous characters, namely, the high-frequency variability and the low abundances toward the end of its range. The percentage of D. triradiatus $[$ D.tri. $\%=$ D.tri. $\cdot 100 /($ D.bro. + D.tri.)] shows the well-established (Backman and Shackleton, 1983) and characteristic rise in relative abundance shortly below its extinc-

\footnotetext{
'Berger, W.H., Kroenke, L.W., Mayer, L.A., et al., 1993. Proc. ODP, Sci. Results, 130: College Station, TX (Ocean Drilling Program).

${ }^{2}$ Department of Geology and Geochemistry, Stockholm University, S-106 91 Stockholm, Sweden.

${ }^{3}$ Godwin Laboratory, Cambridge University, Free School Lane, Cambridge CB2 3RS, United Kingdom.
}

tion level. This rise falls between 42.8 and $43.8 \mathrm{mbsf}$. The age of this event has been estimated to $2.03 \mathrm{Ma}$, as derived from DSDP Site 607 (Raymo et al., 1989).

To illustrate the uppermost part of the ranges of $D$. brouweri and $D$. triradiatus better, their abundance variations were replotted and shown on an expanded scale (Fig. 2). The interval of drastically reduced abundances encompasses about $2.5 \mathrm{~m}$ and ends before the final peak of $D$. brouweri (39.50-39.90 mbsf). The mutual extinction of $D$. brouweri and $D$. triradiatus is interpreted to occur between $39.20 \mathrm{mbsf}(0.0 \mathrm{D}$. brouweri specimens per mm square) and 39.50 mbsf ( 14.9 specimens) (Table 1). These events are considered to have an age of $1.89 \mathrm{Ma}$, about $10 \mathrm{k.y}$. older than the Olduvai/Matuyama boundary (Backman and Shackleton, 1983). The two spikes representing $100 \%$ D. triradiatus that lie above the interpreted extinction level represent two separate occurrences of single specimens, which are thus considered as reworked.

\section{Discoaster pentaradiatus, Discoaster surculus, and Discoaster asymmetricus}

The abundance pattern of $D$. pentaradiatus provides a comparatively distinct disappearance level, somewhere between 53.5 and 53.8 mbsf (Fig. 3). Thus, this short interval is estimated to be about 2.38 m.y. old.

The upper ranges of $D$. surculus and $D$. asymmetricus extend nearly as high as that of $D$. brouweri. The biostratigraphic information the two former species potentially possess is blurred by the anomalously extended ranges, because their extinctions are normally observed below that of $D$. pentaradiatus rather than close to that of $D$. brouweri. It is noteworthy that not a single specimen of Discoaster tamalis has been observed in the counted interval.

\section{REFERENCES}

Backman, J., and Pestiaux, P., 1987. Pliocene Discoaster abundance variations, Deep Sea Drilling Project Site 606: biochronology and paleoenvironmental implications. In Ruddiman, W.F., Kidd, R.B., et al., Init. Repts. DSDP, 94, Pt. 2: Washington (U.S. Govt. Printing Office), 903-910.

Backman, J., Pestiaux, P., Zimmerman, H.B., and Hermelin, O., 1986. Pliocene palaeoclimatic and palaeo-oceanographic development in the North Atlantic: Discoaster abundance and coarse fraction data. In Summerhayes, C.P., and Shackleton, N.J. (Eds.), North Atlantic Palaeoceanography. Spec. Publ., Geol. Soc. London, 21:231-241.

Backman, J., and Shackleton, N.J., 1983. Quantitative biochronology of Pliocene and Pleistocene calcareous nannofossils from the Atlantic, Indian and Pacific oceans. Mar. Micropaleontol., 8:141-170.

Bukry, D., 1973. Low-latitude coccolith biostratigraphic zonation. In Edgar, N.T., Saunders, J.B., et al., Init. Repts. DSDP, 15: Washington (U.S. Govt. Printing Office), 685-703.

Chepstow-Lusty, A., 1990. Nannoplankton as indications of climatic variability in the upper Pliocene [Ph.D. thesis]. Cambridge Univ.

Chepstow-Lusty, A., Backman, J., and Shackleton, N.J., 1989. Comparison of upper Pliocene Discoaster abundance variations from North Atlantic Sites $552,607,658,659$ and 662: further evidence for marine plankton responding to orbital forcing. In Ruddiman, W.F., Sarnthein, M., et al., Proc. ODP, Sci. Results, 108: College Station, TX (Ocean Drilling Program), 121-141. 
1991. Palaeoclimatic control of upper Pliocene Discoaster assemblages in the North Atlantic. J. Micropaleontol., 9:133-143.

Haq, B.U., and Lohmann, G.P., 1986. Early Cenozoic calcareous nannoplankton biogeography of the Atlantic Ocean. Mar. Micropaleontol., 1:119-194.

Martini, E., 1971. Standard Tertiary and Quaternary calcareous nannoplankton zonation. In Farinacci, A. (Ed.), Proceedings of the Second International Conference on Planktonic Microfossils, Roma: Rome (Ed. Tecnoscienza), 739-785.
Raymo, M.E., Ruddiman, W.F., Backman, J., Clement, B.M., and Martinson, D.G., 1989. Late Pliocene variation in Northern Hemisphere ice sheets and North Atlantic deep circulation. Paleoceanography, 4:413-446.

Date of initial receipt: 27 November 1991

Date of acceptance: 11 March 1992

Ms 130B-057

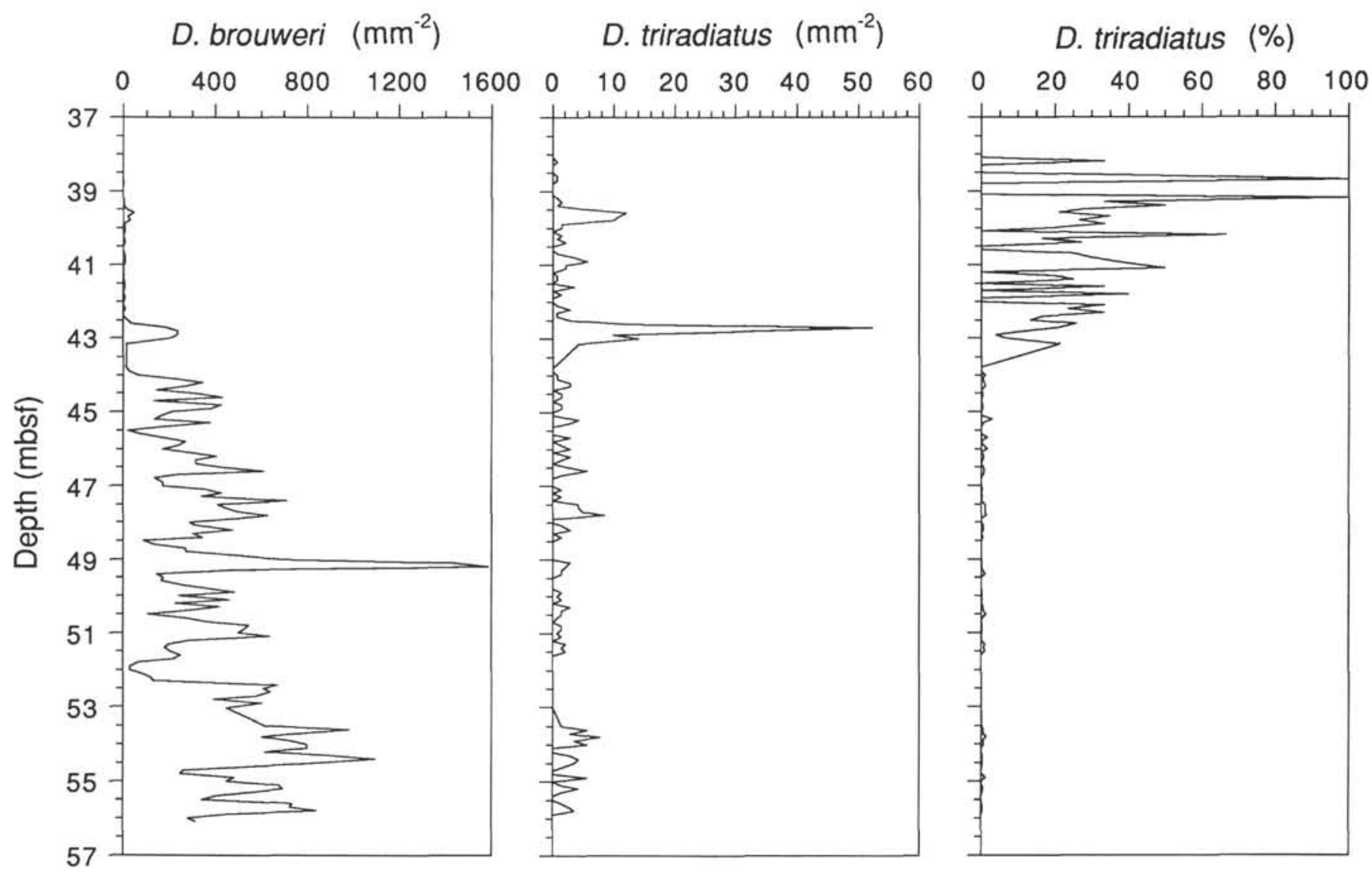

Figure 1. Abundances of D. brouweri and D. triradiatus plotted vs. depth, Hole 806C. 


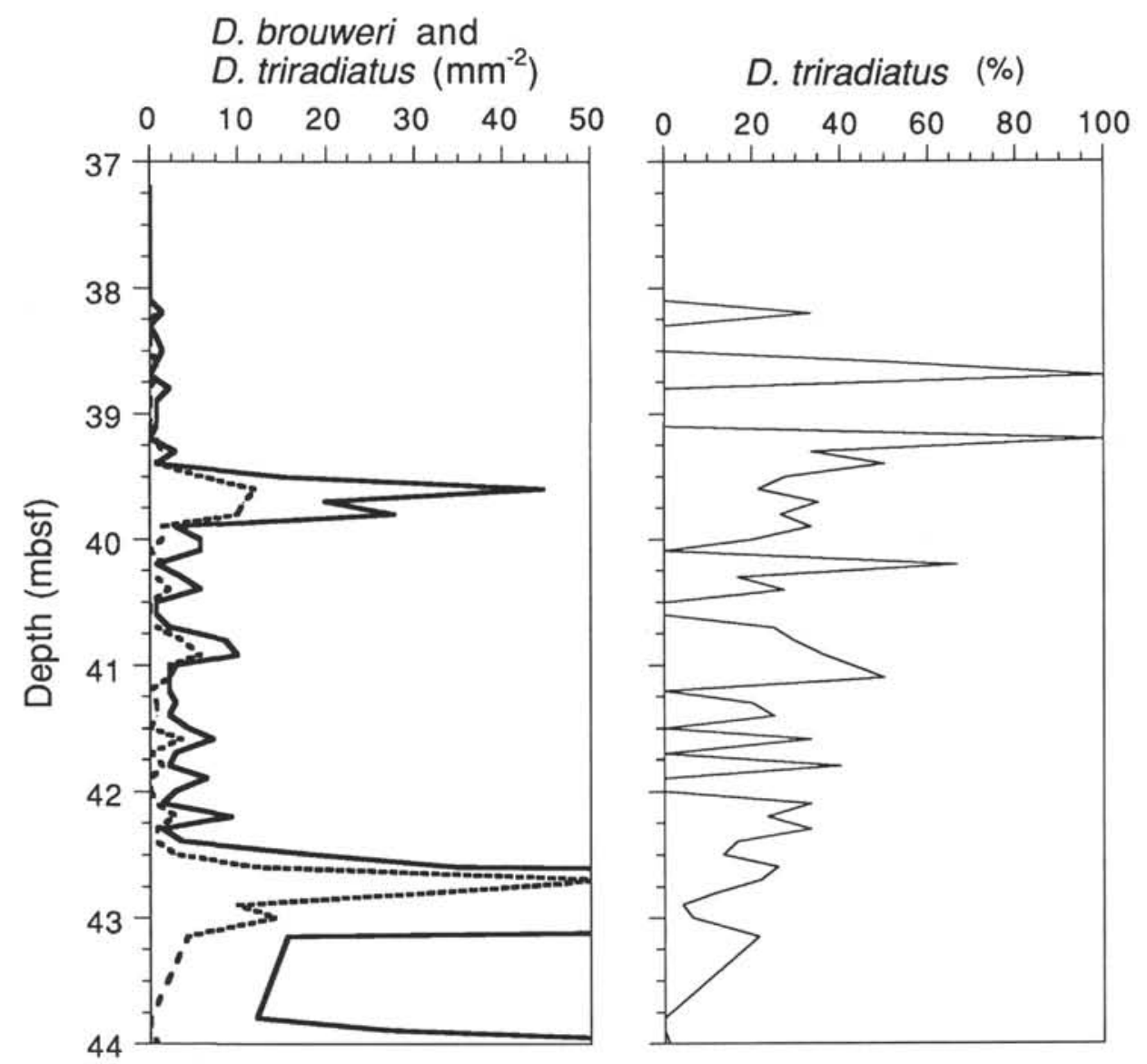

Figure 2. Plots of upper parts of ranges of $D$. brouweri and $D$. triradiatus. Solid bold line $=D$. brouweri $\left(\mathrm{mm}^{-2}\right)$ and dashed bold line $=D$. triradiatus $\left(\mathrm{mm}^{-2}\right)$. 
Table 1. Abundances of Discoaster species in Hole 806C.

\begin{tabular}{|c|c|c|c|c|c|c|c|c|}
\hline $\begin{array}{l}\text { Depth } \\
\text { (mbsf) }\end{array}$ & D. bro. & D. $t r i$. & $\begin{array}{l}\text { D. } t r i . \\
(\%)\end{array}$ & D. pen. & D. sur. & D. asym. & $\begin{array}{l}\text { Depth } \\
\text { (mbsf) }\end{array}$ & D. bro. \\
\hline 37.20 & 0.0 & 0.0 & 0.0 & 0.0 & 0.0 & 0.0 & 46.80 & 137.4 \\
\hline 37.30 & 0.0 & 0.0 & 0.0 & 0.0 & 0.0 & 0.0 & 46.90 & 172.8 \\
\hline 37.40 & 0.0 & 0.0 & 0.0 & 0.0 & 0.0 & 0.0 & 47.00 & 174.9 \\
\hline 37.50 & 0.0 & 0.0 & 0.0 & 0.0 & 0.0 & 0.0 & 47.10 & 356.8 \\
\hline 37.60 & 0.0 & 0.0 & 0.0 & 0.0 & 0.0 & 0.0 & 47.20 & 429.1 \\
\hline 37.70 & 0.0 & 0.0 & 0.0 & 0.0 & 0.0 & 0.0 & 47.30 & 342.7 \\
\hline 37.90 & 0.0 & 0.0 & 0.0 & 0.0 & 0.0 & 0.0 & 47.40 & 713.7 \\
\hline 38.00 & 0.0 & 0.0 & 0.0 & 0.0 & 0.0 & 0.0 & 47.50 & 410.6 \\
\hline 38.10 & 0.0 & 0.0 & 0.0 & 0.0 & 0.0 & 0.0 & 47.60 & 444.6 \\
\hline 38.20 & 1.4 & 0.7 & 33.3 & 0.0 & 0.0 & 0.0 & 47,70 & 499.9 \\
\hline 38.30 & 0.0 & 0.0 & 0.0 & 0.0 & 0.0 & 0.0 & 47.80 & 627.3 \\
\hline 38.40 & 0.7 & 0.0 & 0.0 & 0.0 & 0.0 & 0.0 & 47.90 & 488.5 \\
\hline 38.50 & 1.4 & 0.0 & 0.0 & 0.0 & 0.0 & 0.0 & 48.00 & 290.3 \\
\hline 38.59 & 0.7 & 0.7 & 50.0 & 0.0 & 0.0 & 0.0 & 48.07 & 308.7 \\
\hline 38.70 & 0.0 & 0.7 & 100.0 & 0.0 & 0.0 & 0.0 & 48.20 & 475.8 \\
\hline 38.80 & 2.1 & 0.0 & 0.0 & 0.0 & 0.0 & 0.0 & 48.30 & 305.2 \\
\hline 38.90 & 0.7 & 0.0 & 0.0 & 0.0 & 0.0 & 0.0 & 48.40 & 344.1 \\
\hline 39.00 & 0.7 & 0.0 & 0.0 & 0.0 & 0.0 & 0.0 & 48.50 & 87.1 \\
\hline 39.10 & 0.7 & 0.0 & 0.0 & 0.0 & 0.0 & 0.7 & 48.60 & 131.0 \\
\hline 39.20 & 0.0 & 0.7 & 100.0 & 0.0 & 0.0 & 0.0 & 48.70 & 269.8 \\
\hline 39.30 & 2.8 & 1.4 & 33.3 & 0.0 & 0.0 & 0.0 & 48.80 & 273.3 \\
\hline 39.40 & 0.7 & 0.7 & 50.0 & 0.0 & 0.0 & 0.0 & 48.90 & 532.4 \\
\hline 39.50 & 14.9 & 5.7 & 27.6 & 0.0 & 0.0 & 0.0 & 49.00 & 687.5 \\
\hline 39.60 & 44.6 & 12.0 & 21.3 & 1.4 & 0.0 & 0.0 & 49.10 & 1438.7 \\
\hline 39.70 & 19.8 & 10.6 & 34.9 & 0.0 & 0.7 & 0.0 & 49.20 & 1586.6 \\
\hline 39.80 & 27.6 & 9.9 & 26.4 & 0.0 & 0.0 & 0.0 & 49.30 & 489.2 \\
\hline 39.90 & 2.8 & 1.4 & 33.3 & 0.0 & 0.0 & 0.0 & 49.40 & 146.6 \\
\hline 40.00 & 5.7 & 1.4 & 20.0 & 0.0 & 0.0 & 0.0 & 49.50 & 174.9 \\
\hline 40.09 & 5.7 & 0.0 & 0.0 & 0.0 & 0.0 & 0.0 & 49.59 & 165.0 \\
\hline 40.20 & 0.7 & 1.4 & 66.7 & 0.0 & 0.0 & 0.0 & 49.70 & 255.6 \\
\hline 40.30 & 3.5 & 0.7 & 16.7 & 0.0 & 0.0 & 0.0 & 49.80 & 379.5 \\
\hline 40.40 & 5.7 & 2.1 & 27.3 & 0.0 & 0.0 & 0.0 & 49.90 & 485.0 \\
\hline 40.50 & 0.7 & 0.0 & 0.0 & 0.0 & 0.7 & 0.0 & 50.00 & 241.4 \\
\hline 40.60 & 0.7 & 0.0 & 0.0 & 0.0 & 0.0 & 0.0 & 50.10 & 465.2 \\
\hline 40.70 & 2,1 & 0.7 & 25.0 & 0.7 & 0.0 & 0.7 & 50.20 & 228.0 \\
\hline 40.80 & 8.5 & 3.5 & 29.4 & 0.0 & 0.0 & 0.0 & 50.30 & 419.8 \\
\hline 40.92 & 9.9 & 5.7 & 36.4 & 0.0 & 0.0 & 0.0 & 50.40 & 259.1 \\
\hline 41.00 & 2.8 & 2.1 & 42.9 & 0.0 & 0.0 & 0.0 & 50.50 & 107.6 \\
\hline 41.10 & 2.1 & 2.1 & 50.0 & 0.0 & 0.0 & 0.0 & 50.60 & 274.0 \\
\hline 41.20 & 2.1 & 0.0 & 0.0 & 0.0 & 0.0 & 0.0 & 50.70 & 371.0 \\
\hline 41.30 & 2.8 & 0.7 & 20.0 & 0.0 & 0.0 & 0.0 & 50.80 & 549.4 \\
\hline 41.40 & 2.1 & 0.7 & 25.0 & 0.0 & 0.0 & 0.0 & 50.90 & 524.6 \\
\hline 41.50 & 4.3 & 0.0 & 0.0 & 0.0 & 0.0 & 0.0 & 51.00 & 502.7 \\
\hline 41.59 & 7.1 & 3.5 & 33.3 & 0.0 & 0.0 & 0.0 & 51.09 & 640.0 \\
\hline 41.70 & 2.8 & 0.0 & 0.0 & 0.0 & 0.0 & 0.0 & 51.20 & 289.6 \\
\hline 41.80 & 2.1 & 1.4 & 40.0 & 0.0 & 0.0 & 0.0 & 51.30 & 199.7 \\
\hline 41.90 & 6.4 & 0.0 & 0.0 & 0.0 & 0.0 & 0.0 & 51.40 & 182.0 \\
\hline 42.00 & 2.8 & 0.0 & 0.0 & 0.0 & 0.0 & 0.0 & 51.50 & 204.6 \\
\hline 42.10 & 1.4 & 0.7 & 33.3 & 0.0 & 0.0 & 0.0 & 51.60 & 249.2 \\
\hline 42.20 & 9.2 & 2.8 & 23.5 & 0.0 & 0.0 & 0.0 & 51.70 & 220.2 \\
\hline 42.30 & 1.4 & 0.7 & 33.3 & 0.0 & 0.0 & 0.0 & 51.80 & 68.0 \\
\hline 42.40 & 3.5 & 0.7 & 16.7 & 0.0 & 0.0 & 0.0 & 51.90 & 30.4 \\
\hline 42.50 & 18.4 & 2.8 & 13.3 & 0.0 & 0.0 & 0.0 & 52.00 & 28.3 \\
\hline 42.60 & 34.7 & 12.0 & 25.8 & 0.0 & 0.0 & 0.0 & 52.10 & 82.8 \\
\hline 42.70 & 186.9 & 52.4 & 21.9 & 0.0 & 0.0 & 1.4 & 52.20 & 119.7 \\
\hline 42.80 & 237.9 & 32.6 & 12.0 & 0.0 & 1.4 & 4.3 & 52.30 & 128.2 \\
\hline 42.90 & 236.5 & 9.9 & 4.0 & 0.0 & 0.7 & 1.4 & 52.40 & 673.3 \\
\hline 43.00 & 206.7 & 14.2 & 6.4 & 0.0 & 0.0 & 2.8 & 52.50 & 609.6 \\
\hline 43.15 & 15.6 & 4.3 & 21.4 & 0.0 & 0.0 & 0.0 & 52.59 & 639.3 \\
\hline 43.80 & 12.0 & 0.0 & 0.0 & 0.0 & 0.0 & 0.7 & 52.70 & 579.9 \\
\hline 43.90 & 26.9 & 0.0 & 0.0 & 0.0 & 0.0 & 0.7 & 52.80 & 392.2 \\
\hline 44.00 & 66.6 & 0.7 & 1.1 & 0.0 & 0.7 & 0.0 & 52.90 & 603.9 \\
\hline 44.10 & 215.2 & 0.7 & 0.3 & 0.0 & 1.4 & 0.0 & 53.02 & 448.9 \\
\hline 44.20 & 346.9 & 2.8 & 0.8 & 0.0 & 0.0 & 2.1 & 53.50 & 619.5 \\
\hline 44.30 & 269.0 & 2.8 & 1.0 & 0.0 & 0.7 & 0.0 & 53.60 & 980.6 \\
\hline 44.40 & 143.0 & 0.0 & 0.0 & 0.0 & 0.0 & 0.0 & 53.70 & 773.1 \\
\hline 44.50 & 315.8 & 1.4 & 0.5 & 0.0 & 1.4 & 0.0 & 53.80 & 602.5 \\
\hline 44.60 & 431.9 & 1.4 & 0.3 & 0.0 & 1.4 & 0.0 & 53.90 & 731.4 \\
\hline 44.70 & 134.5 & 0.0 & 0.0 & 0.0 & 0.0 & 0.0 & 54.00 & 798.6 \\
\hline 44.80 & 426.2 & 1.4 & 0.3 & 0.0 & 0.7 & 4.3 & 54.10 & 800.8 \\
\hline 44.90 & 380.9 & 1.4 & 0.4 & 0.0 & 1.4 & 0.7 & 54.20 & 617.4 \\
\hline 45.00 & 212.4 & 0.0 & 0.0 & 0.0 & 0.0 & 0.0 & 54.30 & 885.7 \\
\hline 45.09 & 165.7 & 0.0 & 0.0 & 0.0 & 0.0 & 4.3 & 54.40 & 1094.6 \\
\hline 45.20 & 137.4 & 4.3 & 3.0 & 0.0 & 0.0 & 0.0 & 54.50 & 857.4 \\
\hline 45.30 & 379.5 & 2.8 & 0.7 & 0.0 & 0.0 & 0.0 & 54.70 & 261.3 \\
\hline 45.40 & 171.3 & 0.0 & 0.0 & 0.0 & 0.0 & 0.0 & 54.80 & 249.9 \\
\hline 45.50 & 22.7 & 0.0 & 0.0 & 0.0 & 0.0 & 0.7 & 54.90 & 484.3 \\
\hline 45.60 & 73.6 & 0.0 & 0.0 & 0.0 & 0.0 & 0.0 & 55.00 & 448.2 \\
\hline 45.70 & 172.0 & 2.8 & 1.6 & 0.0 & 0.0 & 1.4 & 55.10 & 677.6 \\
\hline 45.80 & 271.9 & 0.0 & 0.0 & 0.0 & 0.7 & 0.0 & 55.20 & 693.1 \\
\hline 45.90 & 242.1 & 1.4 & 0.6 & 0.0 & 0.7 & 0.0 & 55.30 & 569.9 \\
\hline 46.00 & 173.5 & 2.8 & 1.6 & 0.0 & 0.0 & 1.4 & 55.40 & 405.0 \\
\hline 46.10 & 281.8 & 0.0 & 0.0 & 0.0 & 4.3 & 0.0 & 55.50 & 343.4 \\
\hline 46.20 & 407.8 & 2.8 & 0.7 & 0.0 & 0.0 & 2.8 & 55.60 & 736.3 \\
\hline 46.30 & 315.8 & 1.4 & 0.5 & 0.0 & 0.0 & 0.0 & 55.70 & 725.7 \\
\hline 46.40 & 318.6 & 0.0 & 0.0 & 0.0 & 0.7 & 0.0 & 55.80 & 841.8 \\
\hline 46.50 & 427.6 & 2.8 & 0.7 & 0.0 & 0.0 & 0.7 & 55.90 & 445.3 \\
\hline 46.59 & 611.7 & 5.7 & 0.9 & 0.0 & 1.4 & 0.0 & 56.00 & 283.9 \\
\hline 46.70 & 232.2 & 1.4 & 0.6 & 0.0 & 0.7 & 0.0 & 56.09 & 315.8 \\
\hline
\end{tabular}

Notes: Abundance is expressed as the number of specimens per square millimeter. One (1) counted specimen represents about 0.7 specimens per mm ${ }^{-2}, D . b r o,=D . b r o u w e r i, D . t r i, ~=D . t r i r a d i a t u s, ~ D$. tri. $(\%)=$ percent of $D$. triradiatus relative to $D$. brouweri, $D$. pen. $=D$. pentaradiatus, $D$. sur. $=D$. surculus, and $D$. asym. $=D$. asymmetricus. 

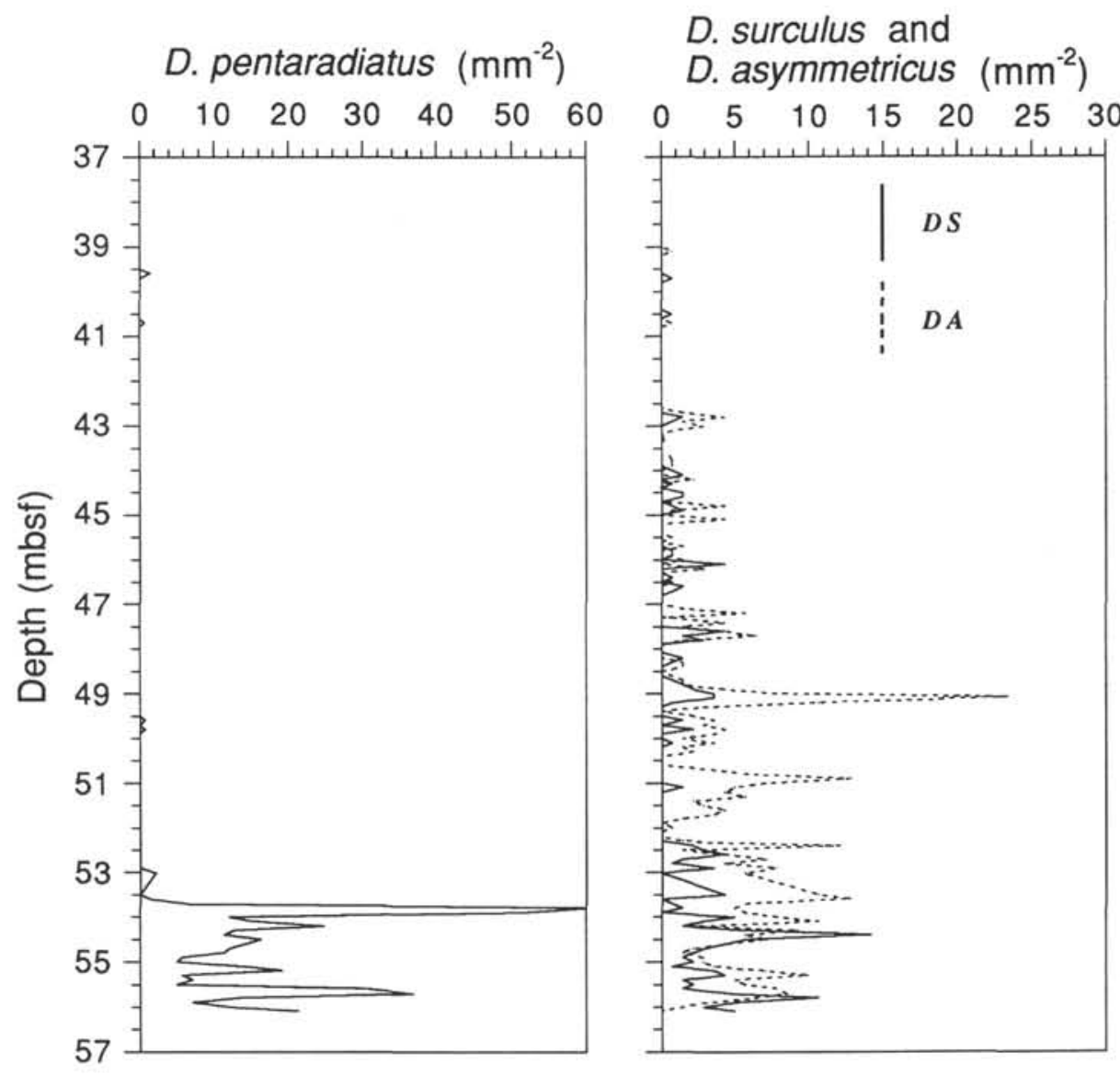

Figure 3. Abundances of D. pentaradiatus, D. surculus (solid line), and D. asymmetricus (dashed line) plotted vs. depth, Hole $806 \mathrm{C}$. 